\title{
Subcutaneous venous port (port A cath) implantation -can be done in a general radiology department as a minimally invasive procedure
}

From: WNDP Chinthaka Appuhamy, MBBS, MD

Department of Diagnostic Radiology

Tan Tock Seng Hospital

11 Jalan Tan Tock Seng

Singapore 308433

\section{Editor:}

The implantable subcutaneous venous access port or Port A cath is a common procedure in patients requiring long-term venous access. It is preferred to external catheters, particularly in patients who receive intermittent long-term infusion therapies, such as chemotherapy, total parenteral nutrition, administration of blood products etc.

Traditionally, port implantation is performed by surgeons under general anesthesia, with venous cut-down in the operation theatre. Image-guided Port A cath insertion by radiologists has following advantages,

- Direct image guided venous access

- Precise catheter tip placement under real time fluoroscopy guidance.

- Minimal complications such as hematoma and pneumothorax formation.

In the authors experience port implantation can be carried out in a general radiology department using real time ultrasound and fluoroscopy guidance with local anesthesia. I describe the technique of trans jugular approach. Right internal jugular vein access (IJV) is preferred in all patients. If the right IJV is occluded, then the left IJV is accessed. In cases of bilateral occlusion, subclavian veins are used. In patients who had undergone mastectomy, the contra lateral IJV was preferred.

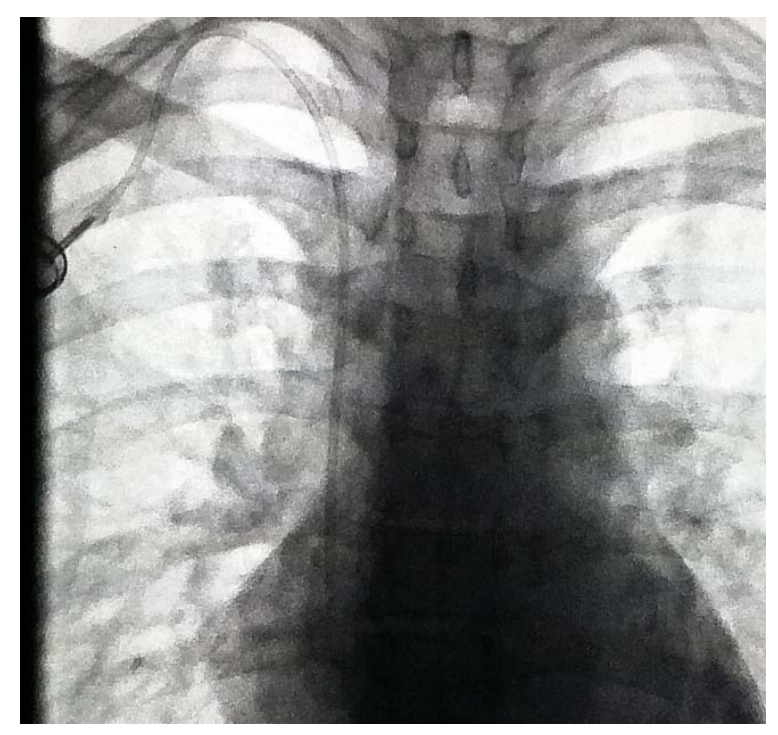

Figure 1: Implanted venous port via right internal jugular vein

\section{Procedure:}

After local anesthetic infiltration venous access is obtained using an $18 \mathrm{G}$ puncture needle or a micropuncture set and a $\mathrm{J}$ wire is inserted and parked in the IVC. $3-3.5 \mathrm{~cm}$ incision is made (size differ according to the brand and the gauge of the catheter- 6.5-8.5 Fr in size) approximately $3 \mathrm{~cm}$ below the clavicle after diluted local anesthetic 


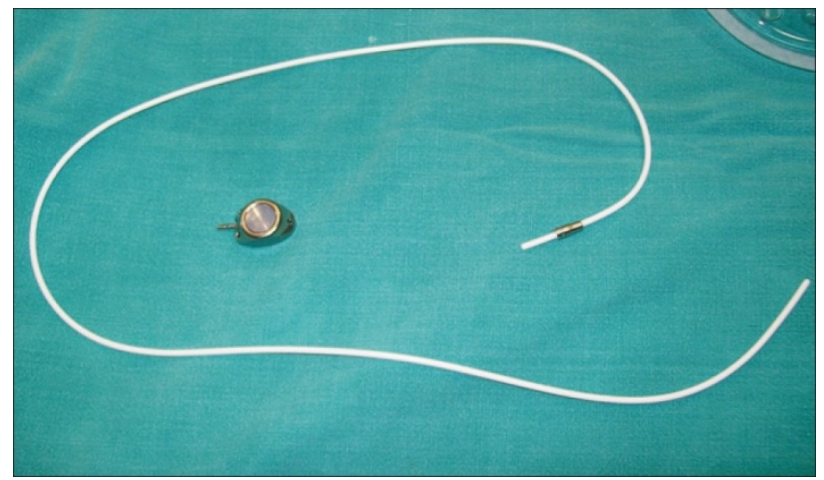

Figure 2: A port and catheter

infiltration. A pocket is created, below or above the incision (above the incision is the authors preference) with the size just enough to accommodate the device. One or two stay sutures can be place to secure the port in to the tissue. The catheter is tunneled through the subcutaneous tract and then inserted into the internal jugular vein using a peel-away sheath (included in the device kit). The catheter tip is positioned in the cavo-atrial junction. The catheter is cut to the size and connect to the port. (Authors preference is to attach the port before inserting the catheter in to the vein). The device is accessed with a non coring Huber needle and flushed with heparin lock solution $(200 \mathrm{iu} / \mathrm{ml})$. Using a non-coring Huber needle, more than 2000 punctures can be made.

Under fluoroscopy, the entire course of the catheter is examined for kinks. The incision closed with interrupted absorbable sutures in the subcutaneous layer to approximate tissues and continuous absorbable suture in the subcuticular layer.

\section{Complications}

Procedural and early complications are quite rare, but they can include hematoma, catheter malposition, arrhythmias, and pneumothorax. Late problems include both thrombotic complications (native venous or
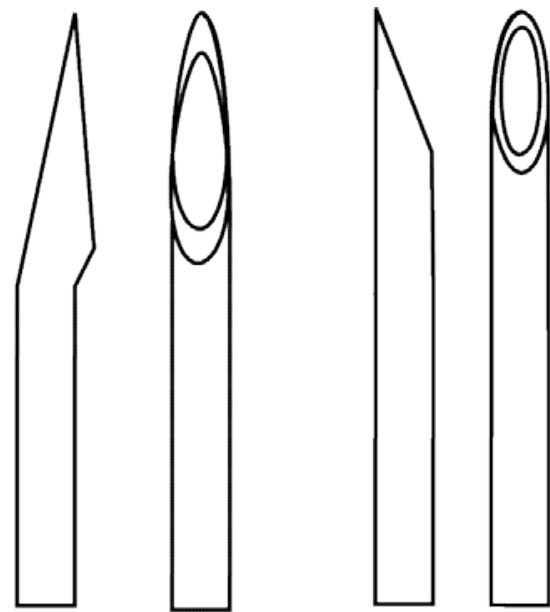

Huber Point Needle Standard Point

port-catheter thrombosis) and infections

Figure 3: Comparison of non coring Huber needle and a normal needle

(tunnel or pocket infections or catheterassociated bloodstream infections). Most guidelines suggest that 0.3 infections/1000 catheter days.

Radiological implantation of subcutaneous venous ports can be performed with similar or lower complication rates as compared to the surgical literature, due to the obvious advantage of imaging guidance.

Hence, we believe that port implantation with imaging guidance will become the preferred implantation method in the future.

\section{References:}

1. Society of interventional radiology quality improvement guidelines for central venous access.

2. Baskin KM, Jimenez RM, Cahill AM, et al. The cavoatrial junction and central venous anatomy: implications for central venous access tip position. J.Vasc Interv Radiol 2008; 19:359-370.

3. Denny DF Jr. Placement and management of long-term central venous access catheters and ports. AJR Am J Roentgenol 1993; 161:385-393. 